\title{
An easy way to increase confidence in beta-amyloid PET evaluation
}

Otakar Belohlavek, Monika Jaruskova

Department of Nuclear Medicine - PET Centre Na Homolce Hospital, Prague, Czech Republic

[Received 11 IV 2017; Accepted 10 V 2017]

\begin{abstract}
BACKGROUND: In patients with brain atrophy, it is not easy to distinguish pathologic uptake of flutemetamol (FMM) in the gray matter from nonspecific, physiologic uptake in the white matter. In this paper we suggest an easy image processing method. MATERIAL AND METHODS: The proof-of-concept study involved three patients with mild cognitive impairment and different graphical findings at FMM-PET. Two-phase FMM-PET was acquired; the early phase represented the perfusion of gray matter, while the late phase depicted the white matter and beta-amyloid load in the gray matter. The border of the gray matter was easily extracted from the early-phase images using thresholding and the isocontour "Edges" color table. The late phase was registered with the edge images of the early phase and displayed using alpha-blending.

RESULTS: Early- and late-phase image fusion displayed with appropriate color tables is presented in three different cases to illustrate the added value of the suggested approach.

CONCLUSIONS: Composite late-phase images with enhanced gray matter borders strongly facilitate assessment of beta-amyloid presence in the gray matter. This is especially helpful in patients with brain atrophy.
\end{abstract}

KEY words: positron-emission tomography, amyloid, brain, dementia, atrophy, flutemetamol

Nucl Med Rev 2017; 20, 2: 107-109

\section{Background}

Specialized training is required for evaluating beta-amyloid PET brain scans due to the difficulty in distinguishing specific, pathologic uptake of ${ }^{18} \mathrm{~F}$-based beta-amyloid seeking tracers (flutemetamol, florbetaben, florbetapir) in the gray matter from nonspecific, physiologic uptake in the white matter. This is especially difficult in the presence of brain atrophy, when the gray matter ribbon is thin and the sulci are flat. Some authors have reported the possibility of using Pittsburgh compound B [1] and florbetapir [2, 3] for the assessment of brain perfusion; images are acquired several minutes after the administration of the radiotracer, when the signal from the brain mainly represents the perfusion of gray matter. We endeavored to utilize early-phase signal for the delineation of gray matter, and to then superimpose its borders on the late-phase image to facilitate the determination of uptake in the gray matter, which is related to beta-amyloid load. In this paper we suggest an image processing method, which is easy to implement and use.

Correspondence to: doc. MUDr. Otakar Belohlavek, CSc.

Department of Nuclear Medicine - PET Centre Na Homolce Hospital

Roentgenova 37/2, 15030 Prague 5, Czech Republic

Tel: +420257272165

Fax: +420257272 163

E-mail: otakar.belohlavek@homolka.cz

\section{Material and methods}

The proof-of-concept study involved three patients with mild cognitive impairment and different graphical findings at FMM-PET. Patients provided signed, informed consent in the course of routine investigation according to the local standard of care. Approval from the institutional review board or ethics committee was not needed for the present work as images were processed retrospectively and anonymously, without any impact on further patient management.

Non-contrast, low-dose CT was performed for the purpose of attenuation correction. A list mode PET acquisition in the PET/CT scanner (Siemens Biograph 40 TrueV HD) followed at the moment $185 \mathrm{MBq}$ of FMM (Vizamyl, GE HealthCare) was administered. Data were acquired for 8 minutes and rebinned to $4 \times 2$-minute datasets for the purpose of motion check only. The motion-free interval was chosen and early phase images were iteratively reconstructed into a $168 \times 168$ matrix, 3 iterations, 21 subsets, zoom 2, Gaussian filter $2 \mathrm{~mm}$ using attenuation, scatter and PSF correction. After an uptake time of 90 minutes, late-phase data were acquired for 20 minutes and rebinned to $4 \times 5$ minutes, other parameters as stated previously.

Both reconstructed volumes were mutually registered and oriented in the Siemens Multi-Modality Work Place using the 3-D tool. Early-phase images were presented in the "Edges" isocontour color table and the threshold was set to delineate a thin superficial 


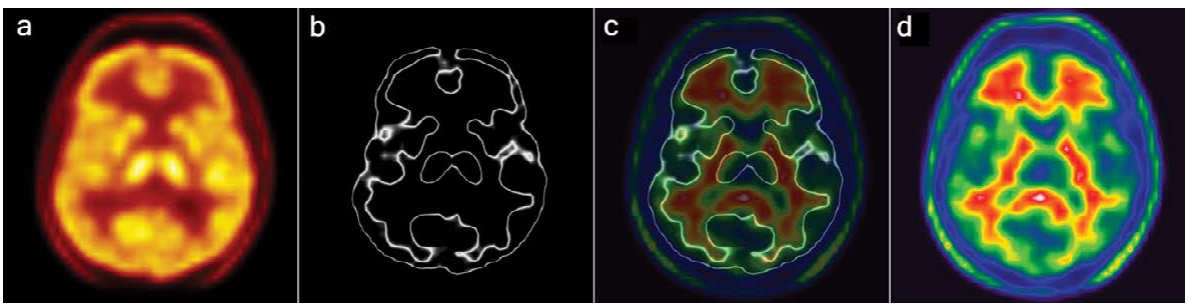

Figure 1. FMM-PET in a 53-year old male with a low pretest probability of dementia. Early-phase image presented in "Hot Body" (A) and "Edges" (B) color tables. There is a normal distribution of FMM in the late phase (D); overlay of images (B) and (D) facilitated the assessment of no pathologic uptake in the gray matter $(\mathrm{C})$. This finding is not consistent with the presence of beta-amyloid

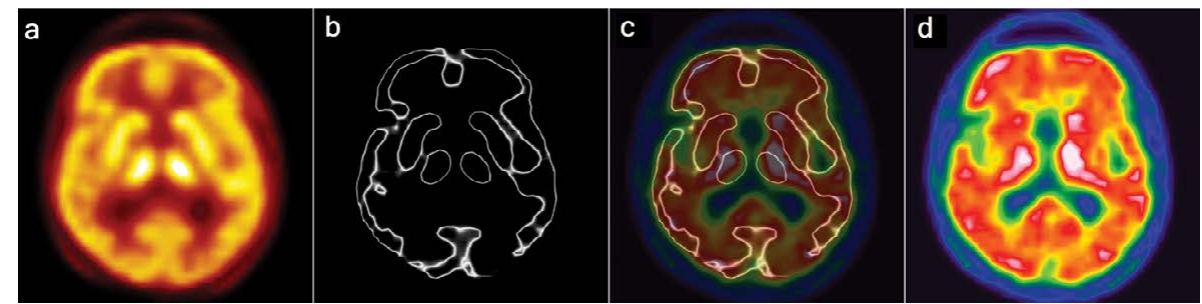

Figure 2. FMM-PET in a 62-year old female with a high pretest probability of Alzheimer disease. Early-phase image presented in "Hot Body" (A) and "Edges" (B) color tables. There is clearly an abnormal distribution of FMM (D) in the late phase; overlay of images (B) and (D) facilitated the assessment of increased uptake in the gray matter $(\mathrm{C})$. This finding is consistent with the presence of beta-amyloid

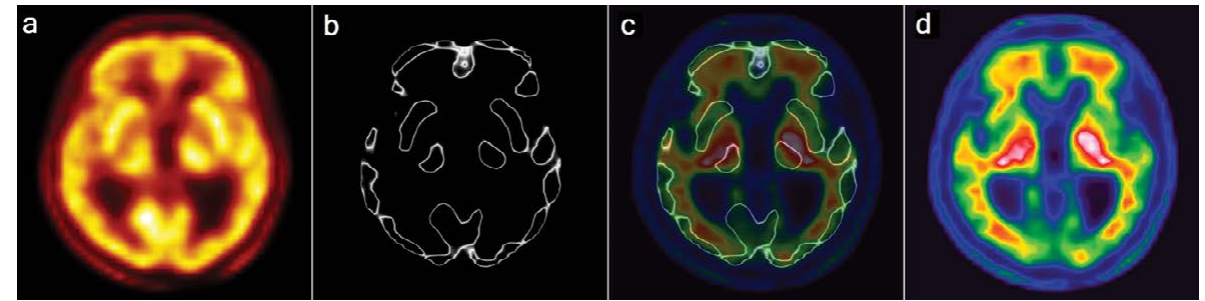

Figure 3. FMM-PET in a 70-year old male with hydrocephalus, brain atrophy and dementia. Early-phase image presented in "Hot Body" (A) and "Edges" (B) color tables. The evaluation of the late phase (D) should be done with respect to the thin gray matter ribbon that makes decision indeterminate, especially in the frontal lobes. Overlay of images (B) and (D) facilitated the demarcation of gray matter and the assessment of no pathologic uptake in the gray matter $(\mathrm{C})$. This finding is not consistent with the presence of beta-amyloid

brain ribbon that mainly represents gray matter. Late-phase images were presented in the "spectrum" color table and the upper threshold was adjusted to $90 \%$ in the pons and cerebellar peduncles. Alpha blending of early-phase edge images with late-phase images was done by manually adjusted the mixing ratio. Beta-amyloid specific uptake in the gray matter was evaluated in critical areas of the frontal and lateral temporal lobes, anterior and posterior cingulum, precuneus and temporo-parietal areas including the insula.

\section{Results}

Visualization of the border between white and gray matter facilitated the assessment of gray matter uptake, enabling the easy distinction between normal, nonspecific FMM uptake in the white matter from pathological uptake in the gray matter, consistent with the presence of beta amyloid. This is illustrated by three different cases:
— in Figure 1, a patient with low pretest dementia probability and normal FMM-PET is presented;

— in Figure 2, a patient with high pretest Alzheimer disease probability and beta-amyloid positivity at FMM-PET is shown;

- the added value of our approach is clearly apparent in a more complicated case of brain hydrocephalus with atrophy, where the gray matter is thin and sulci are flat (Fig. 3).

\section{Discussion}

PET investigation of beta-amyloid performed with an early-phase acquisition may become popular as it represents a "one-stop" complex PET examination of the brain in dementia [4]. The available signal from early-phase FMM-PET can easily be employed in the delineation of the gray matter to help in the assessment of beta-amyloid uptake. We assume similar applicability with other beta-amyloid seeking tracers. The crucial advantage of this approach is the same spatial resolution of both early and late data- 
sets including the same photon spillover from gray to white and white to gray matter; proper thresholding of both studies adequately compensates for spillover and thus facilitates the assessment.

We propose a simple method with the potential to increase confidence in reporting FMM-PET. Validation of improved FMM-PET diagnostic accuracy should be performed once a larger number of early- and late-phase PET datasets with subsequent histological beta-amyloid confirmation become available.

\section{Conflict of interest}

The authors declare that they have no conflict of interest.

\section{Source of funding}

This work was supported by Ministry of Health, Czech Republic - conceptual development of research organization (Nemocnice Na Homolce - NNH, 00023884).

\section{References}

1. Blomquist $\mathrm{G}$, Engler $\mathrm{H}$, Nordberg $\mathrm{A}$, et al. Unidirectional influx and net accumulation of PIB. Open Neuroimag J. 2008; 2: 114-125, doi: 10.2174/ 1874440000802010114, indexed in Pubmed: 19526073.

2. Hsiao IT, Huang CC, Hsieh CJ, et al. Correlation of early-phase 18F-florbetapir (AV-45/Amyvid) PET images to FDG images: preliminary studies. Eur J Nucl Med Mol Imaging. 2012; 39(4): 613-620, doi: 10.1007/s00259-0112051-2, indexed in Pubmed: 22270508.

3. Lin KJ, Hsiao IT, Hsu JL, et al. Imaging characteristic of dual-phase (18) F-florbetapir (AV-45/Amyvid) PET for the concomitant detection of perfusion deficits and beta-amyloid deposition in Alzheimer's disease and mild cognitive impairment. Eur J Nucl Med Mol Imaging. 2016; 43(7): 1304-1314, doi: 10.1007/s00259-016-3359-8, indexed in Pubmed: 27003417.

4. Valentina G, Silvia M, Marco P. Dual-phase amyloid PET: hitting two birds with one stone. Eur J Nucl Med Mol Imaging. 2016; 43(7): 1300-1303, doi: 10.1007/s00259-016-3393-6, indexed in Pubmed: 27105874 